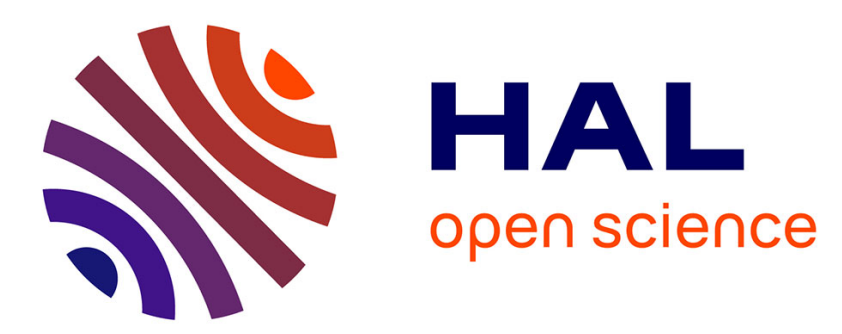

\title{
A Decision-Making approach where Argumentation added value tackles Social Choice deficiencies
}

Pierre Bisquert, Madalina Croitoru, Christos Kaklamanis, Nikos Karanikolas

\section{To cite this version:}

Pierre Bisquert, Madalina Croitoru, Christos Kaklamanis, Nikos Karanikolas. A Decision-Making approach where Argumentation added value tackles Social Choice deficiencies. Progress in Artificial Intelligence, 2019, 8 (2), pp.229-239. 10.1007/s13748-019-00173-3 . lirmm-02180318

\section{HAL Id: lirmm-02180318 https://hal-lirmm.ccsd.cnrs.fr/lirmm-02180318}

Submitted on 11 Jul 2019

HAL is a multi-disciplinary open access archive for the deposit and dissemination of scientific research documents, whether they are published or not. The documents may come from teaching and research institutions in France or abroad, or from public or private research centers.
L'archive ouverte pluridisciplinaire $\mathbf{H A L}$, est destinée au dépôt et à la diffusion de documents scientifiques de niveau recherche, publiés ou non, émanant des établissements d'enseignement et de recherche français ou étrangers, des laboratoires publics ou privés. 


\title{
A Decision-Making approach where Argumentation added value tackles Social Choice deficiencies
}

\author{
Pierre Bisquert*1, Madalina Croitoru ${ }^{\dagger 2}$, Christos Kaklamanis ${ }^{\ddagger 3}$ and \\ Nikos Karanikolas ${ }^{\S 4}$ \\ ${ }^{1}$ IATE, INRA, GraphIK, Montpellier, France \\ ${ }^{2}$ GraphIK, LIRMM, University of Montpellier, Montpellier, France \\ ${ }^{3}$ Computer Technology Institute and Press "Diophantus" (CTI) \& \\ Department of Computer Engineering and Informatics, University \\ of Patras, Patras, Greece \\ ${ }^{4}$ Department of Computer Engineering and Informatics, University \\ of Patras, Patras, Greece
}

\begin{abstract}
Collective decision-making in multi-agents systems is classically performed by employing social choice theory methods. Each member of the group (i.e., agent) expresses preferences as a (total) order over a given set of alternatives, and the group's aggregated preference is computed using a voting rule. Nevertheless, classic social choice methods do not take into account the rationale behind agents' preferences. Our research hypothesis is that a decision made by a group of participants understanding the qualitative rationale (expressed by arguments) behind each other's preferences has better chances to be accepted and used in practice. Accordingly, in this work, we propose a novel qualitative procedure which combines argumentation with computational social choice for modeling the collective decision-making problem. We show that this qualitative approach produces structured preferences that can overcome major deficiencies that appear in the social choice literature and affect most of the major voting rules. Hence, in this paper we deal with the Condorcet Paradox and the properties of Monotonicity and Homogeneity which are unsatisfiable by many voting rules.
\end{abstract}

Keywords Social choice, Argumentation framework, Collective decision-making, Multi-agent systems

\footnotetext{
* pierre.bisquert@inra.fr

$\dagger$ Madalina.Croitoru@lirmm.fr

¥kakl@ceid.upatras.gr

§nkaranik@ceid.upatras.gr
} 


\section{Introduction}

Taking collective decisions is a part of our everyday life. From the simplest ones, e.g., choosing which movie we are going to watch in the theater, to the most complex ones, e.g., selecting a government, a collective decision has to be made. The way to achieve a decision that satisfies the group members though can be a very complex task. It is plausible to wonder what is the rationale behind a decision in addition to the decision itself. Usually the involved participants (decision makers) take their decisions based on their preferences which can be expressed by different viewpoints, criteria and aspects that they consider to be important. One should wonder then what happens when we want to take a justified group's decision where the reasoning of the preferences is clear and the decision should be as fair as possible. This leads us to the following questions.

- How do agents form their thoughts and justify their preferences?

- How should we aggregate them in order to have a "democratic" collective decision?

That are the problems we are dealing with in this paper.

The commonly used way of making a collective decision is using social choice theory and aggregation methods. Each agent of the group expresses her preference as a total order over a set of alternatives, and then the group's preference is computed from the individual preferences using a voting rule. The original motivation of social choice theorists was to model, analyse and give solutions to political decision making in groups of people, but nowadays its basic principles are used in modelling and analysing the kinds of interaction taking place in multi-agent systems. In classical voting the collective decision is computed from quantitative methods by taking into account only the agents' preferences without knowing why the agents have these preferences and what is the rationality behind it. We refer the reader to the Handbook of Computational Social Choice [12] for an analytical description of the problem and the classical voting methods used in the literature. Thus, classical social choice presents a framework where the justifications for the agents' preferences are not considered.

In order to tackle the previously mentioned questions, we believe that decision support systems based on qualitative methods, where the agents understand the rationale behind preferences, have better chances to be accepted by the decision makers. This gives us the motivation to propose a qualitative decisionaiding process for multi-agent systems which combines argumentation [7, 15] with computational social choice [12]. We believe that enriching the collective decision-making procedure with an argumentation framework can benefit the procedure in a twofold way. First, given that agents present justifications for their preferences, it can provide the reasoning behind the decision and secondly, it can model the deliberation phase prior to voting for taking a group decision. Modelling the deliberation phase when agents form their preferences with an argumentation framework permits us to compute extensions, i.e, the collective viewpoint of the group, which construct a preference profile that is "justified". 
Hence, the justified preference profile can be seen as a type of structured profile which is the outcome of a pre-voting phase that consists of a deliberation procedure. Consequently, this gives us the motivation to study in this paper a decision-making approach based on argumentation, but also on social choice which provides the means to aggregate the structured outcome, i.e., the justified preference profile.

In our approach we are going to place the decision problem within the boundaries of an abstract argumentation framework. Abstract argumentation theory is easy to understand and provides a robust tool for non-monotonic reasoning. It was first introduced by Dung in 1995 [15] and is based on the construction, the exchange and the evaluation of interacting arguments. The argumentation systems are modelled by graphs, where the nodes represent the arguments, and the edges represent the attacks between them. Various semantics defined by Dung and other researchers have been proposed to identify the acceptability of sets of arguments, which are based on the attack relations between them.

In the problem considered in this paper, the decision to be recommended lies on a set of alternative options, which will be referred in the reminder of the paper as alternatives. The decision will be derived from the justified preferences of the set of agents over the set of the alternatives. The justified preferences are the outcome of a debate phase (deliberation) where each agent reveals her preferences by providing a ranking over the alternatives and a justification for this ranking. The collection of agents' rankings is known as preference profile. The preference profile of the agents along with the justifications are used to build the arguments and then the argumentation framework will help us build the justified preference profile which includes the preferences produced after the deliberation and corresponds to the different collective viewpoints of the agents. The objective is to fairly aggregate the justified viewpoints of the agents and hence, the justified preference profile is reported to a voting rule, which then singles out the winning alternative and the ranking of the remaining ones.

The classical problem in social choice theory is which voting rule is the most appropriate for aggregating the preference profile. Unfortunately, due to two fundamental impossibility results from social choice theory there is no hope of finding a voting rule that can be "perfect". The first one was imposed by Arrow in 1950 [5] and the second one by Gibbard in 1973 and Satterthwaite in 1975 [21, 29]. Due to these results we know some vital criteria cannot be satisfied all at the same time. Despite that, social choice theory has enhanced our perception among proposed voting rules, where each of them has different characteristics, qualities, and weaknesses but all of them have the same goal, i.e., to elect the fairest socially outcome. It should be noted here that each rule has some assumptions of what is the fairest outcome. For example, when there are two alternatives and an odd number of agents, the majority rule is unanimously considered a perfect, in terms of fairness, method of selecting the winner. However, when we have three alternatives or more, majority rule is not appropriate anymore and another rule should be used. One of the most prominent rules in the history of social choice which is generally acclaimed as a 
founding method of the field is the one proposed by the Marquis de Condorcet in 1785 [13], and bears his name. The Condorcet method relies on comparisons between each pair of alternatives and the winner of the election, which is known as the Condorcet winner, reflects the best choice for the social good according to social choice theorists. An alternative $x$ is said to beat alternative $y$ in a pairwise election comparison) if a majority of agents prefer $x$ to $y$, i.e., rank $x$ above $y$. The alternative who beats every other alternative in a pairwise comparison is the winner of the election. However, the Condorcet method has a major weakness as there are preference profiles where the Condorcet winner does not exist. This problem arises when the preferences of the majority are cyclic, i.e., not transitive. For example, if we have three alternatives $A, B, C$ and the results of the pairwise comparisons are: $A$ beats $B, B$ beats $C$ and $C$ beats $A$, then we say that a voting cycle occurs. This contradictory phenomenon is known as the Condorcet paradox as defined by Black in 1958 [8]. Despite this paradox, the Condorcet criterion is widely acclaimed as one of the most intuitive ways of voting and will be used to aggregate the justified preferences. To strengthen this perspective, we show that our method always provides justified preference profiles where the Condorcet paradox does not occur.

In order to evaluate the quality of well-known voting rules social choice theorists have defined properties that should be satisfied from a social point of view. Unfortunately, there are well-known voting rules that fail to satisfy major "socially desirable" properties. We can mention here, for example, Dodgson's voting rule who fails to satisfy the properties of monotonicity and homogeneity $[19,11]$. These properties are considered by social choice theorists to be extremely basic; a voting rule is monotonic if it is indifferent to pushing a winning alternative upwards in the preferences of the agents, and homogeneous if it is invariant under duplication of the electorate. Apart from Dodgson's rule, other important rules, i.e., Plurality with Runoff, Alternative Vote (Instant Runoff Voting), Nanson's and Coombs', also fail to satisfy monotonicity [18].

Our work Our research is driven from use-cases where the objective is to reach a unanimous or close to a consensus decision among the group (group consensus approach). It is common in this approach that a deliberation phase is conducted among the agents in the group in order to express their preferences and the reasoning (justifications) behind them. We model this group decisionmaking problem with the following proposed method where the framework built from the justifications of the preferences, can lead to extensions where we have a reduced number of ambiguous preferences among the agents.

More specifically, in our technique, we will use the agents' preferences (rankings) and the justifications for those rankings to compute arguments which will be the base of the argumentation framework. These arguments will rely on the justification of the pairwise individual preferences given by each agent. The set of coherent viewpoints, i.e., the set of extensions $\boldsymbol{E}$, will be computed according to the "preferred" semantics and each extension corresponds to a ranking of the alternatives which contains information about the different viewpoints 
of the agents' preferences. Hence, the collection of all the extensions provides the justified preference profile. When there is no consensus among the agents' preferences, various possible extensions exist. Hence, we cannot take a decision based only on the argumentation framework. That leads to the consideration of a classical voting problem which has as input the justified preferences of the agents over the alternatives. The concluding voting problem gives us the motivation to study the following social choice theoretic properties, i.e., the Condorcet paradox, monotonicity and homogeneity. In a nutshell our contribution is as follows. Given the preference profile we show that the construction of the justified preference profile permits a type of structured preferences where the Condorcet paradox can be avoided, as well as other social choice properties can be satisfied. Hence, we can use the Condorcet method to obtain the final ranking.

Discussion The intuition behind the aggregation of the rankings extracted from extensions and the reason for considering them as (virtual) voters, i.e., the justified preference profile, can be seen from both an argumentative and social choice perspective. From an argumentative perspective, an extension can be seen as a consistent possible interpretation of justified preference relations and a subset of the group's collective viewpoint. Hence, the unique ranking argument entailed in an extension can be considered then as a part of the collective decision and hence, the corresponding ranking can be considered as a new individual voter. Therefore, in order to compute a collective decision taking into account all the possible viewpoints of the group, we must apply an aggregation mechanism/voting function on the (virtual) voters extracted from the extensions' rankings. From a social choice perspective, the "justified" votes are related to original preference profile. It is the justifications built in the arguments that define and compute (according to used semantics) the justified preference profile. By using "preferred" semantics in our approach we show that the Condorcet paradox can be avoided and is let for future work to see if other kind of semantics, e.g., adding weights to extensions with regards to the importance of the number of agents supporting the preference relations, can lead to the same results. Concluding, given the two abovementioned intuitive perspectives, we state that our approach leads to an interesting voting problem. It is therefore meaningful to study social choice properties for the input's profile, which is the justified preference profile. Hence, the need to prove the theoretic results included in this paper.

Related work To the best of our knowledge, the application of argumentation into social choice theory with the objective of aiding the decision-making under the social choice perspective is a relatively new domain, however, several work has been done on the combination of each pair of these fields. Decisionmaking has begun evolving from the 60s when Bernard Roy in 1966 [6] and in 1968 [26] introduced the class of ELECTRE methods for aggregating preferences expressed on multiple criteria, which set the foundations of the "outrank- 
ing methods" that were further deployed by Ostanello in 1985 [25] and Roy in 1991 [27]. Decision-making and Social choice theory are two closely correlated fields whose objective is to aggregate the partial preferences into a collective preference. Arrow and Raynaud in 1986 [4] were the first that presented a general exploration of the links between social choice theory and decision-making. Social choice theory has been integrated in the analysis of some popular aggregation methods in multi-criteria decision-making. Let us mention, for example, the ordinal methods in multi criteria decision-making which were developed by Roy [27] and Roy and Bouyssou [28] and are based on the Condorcet method. In addition, scoring voting methods like the Borda count are integrated in the cardinal methods for multi-criteria aggregation, e.g., Keeney and H. Raiffa [23] and Von Winterfeldt and Edwards [30].

Several researchers have proposed the use of argumentation in decisionmaking. The work of Fox and Parsons in 1997 [20] is one of the first works that tried to deploy an argumentative approach to decision-making stating the difference between argumentation for actions and argumentation for beliefs. Most of the argumentation-based approaches objective is to select the best solution (alternative option), e.g., Karacapilidis and Papadias [22] and Morge and Mancarella [24]. On the contrary, in decision-making several different problem statements with different objectives are allowed, i.e., choosing, rejecting, ranking, classifying the set of alternatives. Regarding the aggregation, several approaches like the ones by Amgoud et al. [2] and by Bonnefon and Fargier [10] used procedures based only on the number or the strength of arguments, while in decision-making there have been proposed many aggregation procedures. Another example of work towards this direction is the one by Amgoud and Prade [3] which proposes an abstract argumentation-based framework for decision-making. The model follows a 2-step procedure where at first the arguments for beliefs and options are built and at the second step we have pairwise comparisons of the options using decision principles.

There have been also studied many problems on the intersection of Social choice and Argumentation which are loosely related to our work. Most of the issues in this research area view the problem from an argumentative perspective and deal with collective argumentation. Most of the works refer to the problem of aggregating individual argumentation frameworks to a collective one. The aggregation mechanisms provided to solve the problem rely on social choice, which provides the means to accomplish that. An informative survey on these problems in collective argumentation is provided by Bodanza et al. [9]. This area of research in collective argumentation is not only restricted to finding aggregation mechanisms. For example, in the work of Airiau et al. [1] the goal differentiates and instead they study the computational complexity of a problem defined as the "rationalisability problem". In this problem each agent has its own argumentation framework $A F_{i}$ and the aim is to identify if there are a single master argumentation framework $A F$, an association of arguments with values and a profile of preference orders over values that can "explain" all the $A F_{i}$ (i.e, $A F$ rationalizes the set of $A F_{i}$ ). 
There are a number of ways for formalizing the concept of reasoning. Hence, the reasoning behind agents' preferences has also been investigated from a different perspective than we do. One prominent work on identifying the reasons in preferences is the one by Dietrich and List [14], where they formulate a reasonbased theory of rational choice in which agents' form preferences according to their motivating reasons. In this work they also clarify the relationship between deliberation for reasons and for rational choices.

\section{Preliminaries}

In the following, we present several notions needed to go further with our approach.

\subsection{Social Choice Theory}

We consider a set of $N=\{1, \ldots, n\}$ agents and a set of alternatives $A,|A|=m$. Each agent $i \in N$ has preference relations $(\succ)$ over the alternatives denoted with $x \succ_{i} y$ which means that agent $i$ prefers alternative $x$ to $y$. We define that each irreflexive preference relation satisfies transitivity antisymmetry and comparability, and hence, the set of all the preference relations for agent $i$ produces a linear (strict total) order $\succ_{i}$ on $A$, i.e., the ranking of agent $i$ over the alternatives. Let $\mathcal{L}_{A}$ be the set of linear orders over $A$. A preference profile $\succ_{P P}=\left\langle\succ_{1}, \ldots, \succ_{n}\right\rangle \in \mathcal{L}_{A}^{n}$ is a collection of the linear orders for all the agents. For each fixed value of $n$, a voting rule is a mapping $f: \mathcal{L}_{A}^{n} \rightarrow 2^{A} \backslash\{\emptyset\}$ from preference profiles to nonempty subsets of alternatives, which designates the winner(s) of the election. For two candidates $x, y \in A$ and $\succ_{P P} \in \mathcal{L}_{A}^{n}$, alternative $x$ beats $y$ in a pairwise comparison if $\left|\left\{i \in N: x \succ_{i} y\right\}\right|>n / 2$, that is, if a (strict) majority of agents prefer $x$ to $y$. The winner according to the Condorcet method is an alternative that beats every other alternative in a pairwise comparison. A Condorcet winner does not always exist due to the following paradox. The Condorcet paradox (also known as voting paradox or the paradox of voting) is a situation in which the collective preference profile can be cyclic (i.e., not transitive), even if the preferences of individual agents are not cyclic. A voting cycle occurs when we have three alternatives $x, y, z$ such that $x$ beats $y, y$ beats $z$ and $z$ beats $x$ in pairwise comparisons.

\subsection{Argumentation}

In order to be general with regards to the deliberation step, we are using the abstract argumentation framework proposed by Dung in 1995 [15]:

Definition[Argumentation framework] An argumentation framework (AF) is a pair $(\mathbf{A}, \mathbf{R})$, where $\mathbf{A}$ is a finite nonempty set of arguments and $\mathbf{R}$ is a binary

relation on $\mathbf{A}$, called attack relation. Let $\mathcal{A}, \mathcal{B} \in \mathbf{A}, \mathcal{A} \mathbf{R} \mathcal{B}$ means that $\mathcal{A}$ attacks $\mathcal{B}$. 
The coherent sets of arguments (called "extensions") are determined according to a given semantics whose definition is usually based on the following concepts:

Definition[Conflict-free set, defense and admissibility] Given an $\operatorname{AF}(\mathbf{A}, \mathbf{R})$, let $\mathcal{A}, \mathcal{B} \in \mathbf{A}$ and $\mathcal{S} \subseteq \mathbf{A}$. We say that

- $\mathcal{S}$ is conflict-free if and only if there do not exist $\mathcal{A}, \mathcal{B} \in \mathcal{S}$ such that $\mathcal{A R} \mathcal{B}$,

- $\mathcal{S}$ defends an argument $\mathcal{A}$ if and only if each argument that attacks $\mathcal{A}$ is attacked by an argument of $\mathcal{S}$, and

- $\mathcal{S}$ is an admissible set if and only if it is conflict-free and it defends all its elements.

Definition[Semantics] Given an $\operatorname{AF}(\mathbf{A}, \mathbf{R})$, let $\mathcal{E} \subseteq \mathbf{A}$. $\mathcal{E}$ is

- a complete extension if and only if $\mathcal{E}$ is an admissible set and every argument which is defended by $\mathcal{E}$ belongs to $\mathcal{E}$,

- a preferred extension if and only if $\mathcal{E}$ is a maximal admissible set (with respect to set inclusion),

- the grounded extension if and only if $\mathcal{E}$ is a minimal (with respect to set inclusion) complete extension, and

- a stable extension if and only if $\mathcal{E}$ is conflict-free and attacks any argument $\mathcal{A} \notin \mathcal{E}$.

Given a semantics $s$, the set of extensions of $(\mathbf{A}, \mathbf{R})$ is denoted by $\boldsymbol{E}_{s}$.

Example 1. Given an $A F(\mathbf{A}, \mathbf{R})$ with $\mathbf{A}=\{\mathcal{A}, \mathcal{B}, \mathcal{C}, \mathcal{D}, \mathcal{E}\}$ and $\mathbf{R}=$ $\{(\mathcal{A}, \mathcal{B}),(\mathcal{B}, \mathcal{A}),(\mathcal{A}, \mathcal{C}),(\mathcal{C}, \mathcal{D}),(\mathcal{D}, \mathcal{E}),(\mathcal{E}, \mathcal{C})\}$

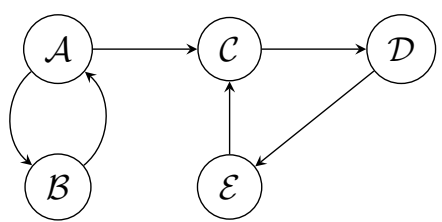

- The complete extensions are \{\}$,\{\mathcal{A}, \mathcal{D}\}$ and $\{\mathcal{B}\}$

- The preferred extensions are $\{\mathcal{A}, \mathcal{D}\}$ and $\{\mathcal{B}\}$

- The unique grounded extension is \{\}

- The stable extension is $\{\mathcal{A}, \mathcal{D}\}$ 
It should be noted that in this paper we want to ensure that each extension represents a full ranking over the alternatives justified by preference relation arguments, which is needed since extensions will be used as (virtual) voters. In order to do that, we focus on the preferred semantics since it ensures to cover all the maximal sets of arguments, which corresponds to the "viewpoints" on the possible rankings of alternatives. Please note that future work will consider the study of other argumentation semantics.

Based on these notions, we can now present the model combining the strengths of social choice and argumentation.

\section{A decision model based on justified prefer- ences}

In the proposed model we are considering the case of taking a collective decision using a qualitative argumentative approach and voting theory, in order to reflect real-life decision problems where a deliberation phase is present. In our problem the input is a set of alternatives as well as the justified preferences of agents over these alternatives. In this paper, each agent provides a justification for each of her preference relations on the alternatives and the preferences are restricted to satisfy a transitive relation so as to allow for the ranking of the alternatives to be built. Observe that the suggested process is an argumentative approach that relies on combining the "qualitative" preferences, which is incomparable to a voting rule whose role is to aggregate the individual preferences with quantitative methods. We use this information to formulate arguments which express the agents' preferences. More precisely, we are going to distinguish between three types of arguments: "preference relation" arguments, "ranking" arguments and "generic" arguments.

Preference relation arguments. A preference relation argument $\mathcal{A}_{x y}$ represents a justification given by an agent to consider the preference $x \succ y$. Note that we may have multiple $\mathcal{A}_{x y}$ arguments, in the case where some agents have different justifications for the preference $x \succ y$. The set of preference relation arguments is denoted $\mathbf{A}_{P R}$.

It should be noted that due to what they represent, the arguments $\mathcal{A}_{x y}$ and $\mathcal{A}_{y x}$ cannot be considered together in a coherent view point since they are "opposed". Consequently, we assume that those arguments are attacking each other.

Example 2. Consider a set of three agents $\{1,2,3\}$ deciding which movie they want to watch at the theater tonight. A collective decision has to be made out of the three movies that are played in the nearest theater, i.e., "Beauty and the beast", "Free fire", "Going in style". We denote the set of the alternatives $\{a, b, c\}$. The preference relations of the agents over $\{a, b, c\}$ and their justifications are the following: 
- $\mathcal{A}_{a b}: a \succ_{1} b$, because a has IMDB rating 7.7 and $b$ has 7.2

- $\mathcal{A}_{b c}: b \succ_{1} c$, because $b$ has IMDB rating 7.2 and $c$ has 6.8

- $\mathcal{A}_{a c}: a \succ_{1} c$, because a has IMDB rating 7.7 and c has 6.8

- $\mathcal{A}_{b a}: b \succ_{2} a$, because $b$ has a higher rating than a in Rotten Tomatoes

- $\mathcal{A}_{b c}^{\prime}: b \succ_{2} c$, because $b$ has a higher rating than $c$ in Rotten Tomatoes

- $\mathcal{A}_{c a}: c \succ_{2}$ a, because $c$ has a higher rating than a in Rotten Tomatoes

- $\mathcal{A}_{a b}^{\prime}: a \succ_{3} b$, because Agent 3 prefers fantasy to action movies

- $\mathcal{A}_{c b}: c \succ_{3} b$, because Agent 3 dislikes action movies

- $\mathcal{A}_{c a}^{\prime}: c \succ_{3}$ a, because Agent 3 prefers horror to fantasy movies

Note that the preferences of the agents are cyclic and trigger the Condorcet paradox: there is a majority of agents for $a \succ b$, another majority for $b \succ c$ and a third majority for $c \succ a$.

We can represent the above arguments in the following graph which is depicted in figure 1:
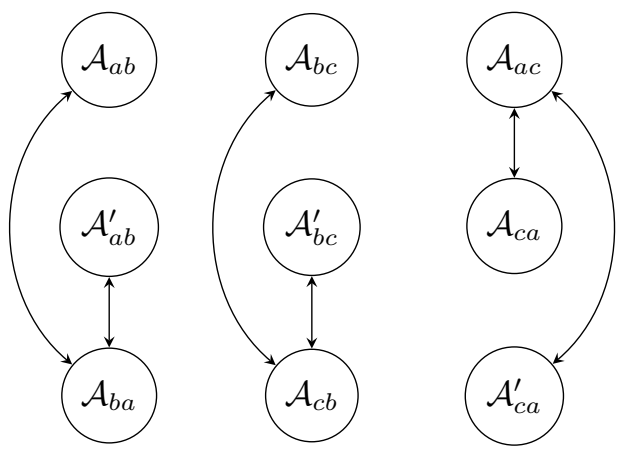

Figure 1: The argumentation graph containing the preference relation arguments and their attacks.

Ranking arguments. A ranking argument represents one of the possible rankings over the considered alternatives. It is important to note that in our setting, we always consider all the possible ranking arguments; it will be the agents' prerogative to justify why a ranking should not be considered as we will see below.

We denote by $\mathbf{A}_{\mathcal{R}}$ the set of all the possible ranking arguments and by $\mathbf{A}_{\mathcal{R}_{x \cdots y}}$ the set of ranking arguments where the preference $x \succ \cdots \succ y$ is satisfied. Moreover, we define a special ranking argument $\mathcal{A}_{B}$ that represents a ranking without preference; it can be seen as the blank vote resulting from either nontransitive preference relations or no justified preferences. 
Like preference relation arguments, we consider ranking arguments as mutually inconsistent. For this reason, we assume that ranking arguments are attacking each other, with the exception of $\mathcal{A}_{B}$ that attacks no argument. In this way, we represent the fact that having a reason to consider a ranking forbids the possibility of considering blank voting.

Furthermore, ranking arguments can be attacked by preference relation arguments. Indeed, giving a justification for $x \succ y$ (i.e., enunciating an argument $\mathcal{A}_{x y}$ ) is a reason for not considering all the rankings with $y \succ x$ (i.e., $\mathbf{A}_{\mathcal{R}_{y x}}$ ); here, $\mathcal{A}_{x y}$ is attacking the elements of $\mathbf{A}_{\mathcal{R}_{y x}}$. Please note that we want the ranking arguments to be justified by preference relation arguments, hence, we do not allow ranking arguments to attack conflicting preference relation arguments. If we allowed this to happen, then the ranking arguments would be able to defend and justify themselves directly (in the context of Dung's abstract argumentation), which is not desirable.

Example 2 (cont.). We complete the previous argumentation framework with the set of ranking arguments $\mathbf{A}_{\mathcal{R}}$ for all the possible permutations of $\{a, b, c\}$ while adding the attacks between the preference relation arguments and the ranking arguments. We obtain:

- $\mathcal{A}_{a b}$ and $\mathcal{A}_{a b}^{\prime}$ attack $\mathcal{A}_{\mathcal{R}_{b a c}}, \mathcal{A}_{\mathcal{R}_{b c a}}$ and $\mathcal{A}_{\mathcal{R}_{c b a}}$

- $\mathcal{A}_{b a}$ attacks $\mathcal{A}_{\mathcal{R}_{a b c}}, \mathcal{A}_{\mathcal{R}_{a c b}}$ and $\mathcal{A}_{\mathcal{R}_{c a b}}$

- $\mathcal{A}_{b c}$ and $\mathcal{A}_{b c}^{\prime}$ attack $\mathcal{A}_{\mathcal{R}_{c a b}}, \mathcal{A}_{\mathcal{R}_{a c b}}$ and $\mathcal{A}_{\mathcal{R}_{c b a}}$

- $\mathcal{A}_{c b}$ attacks $\mathcal{A}_{\mathcal{R}_{b c a}}, \mathcal{A}_{\mathcal{R}_{b a c}}$ and $\mathcal{A}_{\mathcal{R}_{a b c}}$

- $\mathcal{A}_{a c}$ attacks $\mathcal{A}_{\mathcal{R}_{\text {bca }}}, \mathcal{A}_{\mathcal{R}_{c b a}}$ and $\mathcal{A}_{\mathcal{R}_{c a b}}$

- $\mathcal{A}_{c a}$ and $\mathcal{A}_{c a}^{\prime}$ attack $\mathcal{A}_{\mathcal{R}_{a b c}}, \mathcal{A}_{\mathcal{R}_{b a c}}$ and $\mathcal{A}_{\mathcal{R}_{a c b}}$

Generic arguments. Generic arguments regroup all the other possible arguments that can arise during a debate. In particular, those arguments are only able to attack other generic arguments and preference relation arguments (for instance if the reason given for considering $x \succ y$ is itself justified to be wrong).

Example 2 (cont.). During the debate phase new arguments appear. Agent 1 contradicts the information of Agent 2 about alternative $c$ because $c$ has lower rating in Rotten Tomatoes and also contradicts Agent 3 because $c$ is a comedy movie. Hence, we form the following arguments $\mathcal{A}_{1}=(c$ has lower rating than a in rotten tomatoes) and $\mathcal{A}_{2}=\left(c\right.$ is comedy) such that $\mathcal{A}_{1}$ attacks $\mathcal{A}_{c a}$ and $\mathcal{A}_{2}$ attacks $\mathcal{A}_{c a}^{\prime}$.

The following Figure 2 gives a partial representation of the new arguments and attacks. ${ }^{1}$

\footnotetext{
${ }^{1}$ Please note that for the sake of clarity, we are not drawing all the edges in the argumentation graph, but a subset of the edges demonstrating the attacks between preference relation arguments and ranking arguments.
} 


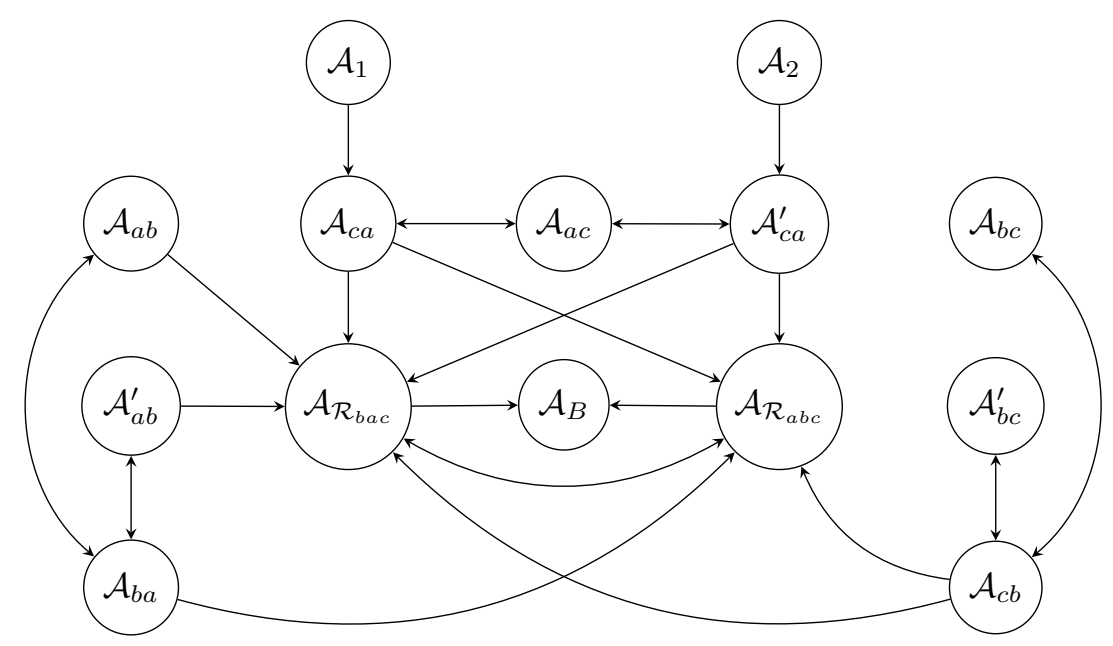

Figure 2: A partial presentation of the argumentation graph for two particular ranking arguments.

It is important to note that while the flexibility offered by the abstract argumentation setting is convenient for its generality, it can also lead to undesirable behaviors. Hence, we propose the following restriction.

Axiom 1 (Independence of preference justifications). Given two preference relation arguments $\mathcal{A}_{x y}$ and $\mathcal{A}_{u v}$, such that $\{x, y\} \neq\{u, v\}$, there is no generic argument $\mathcal{A}_{g}$ such that both paths of attacks from $\mathcal{A}_{g}$ to $\mathcal{A}_{x y}$ and from $\mathcal{A}_{g}$ to $\mathcal{A}_{\text {uv }}$ exist.

The intuition is that the discussions about each pairwise preference are independent, i.e., a generic argument cannot have an impact on preferences over different alternatives. We assume that any generic argument general enough to have an effect on several pairwise comparisons can be separated into "pairwise exclusive" arguments. For instance, an argument rebutting the facts that $x$ is over $y$ and $u$ is over $v$ because of some reason $R$ can be separated into two generic arguments: one attacking $\mathcal{A}_{x y}$ and one attacking $\mathcal{A}_{u v}$ (both because of reason $R$ ).

Computing the justified profile. Using the arguments and attacks shown above in an abstract argumentation framework, called the ranking-completion argumentation framework, it is possible to compute the sets of "coherent preferences", represented by the extensions. ${ }^{2}$ Hence, it is important to remark that

\footnotetext{
${ }^{2}$ Please note that we assume that no odd-length attack cycle may exist between generic arguments in the argumentation framework (such cases would be handled during the actual deliberation). Indeed, allowing the existence of odd-length cycles could lead to the computation of an empty extension which is not a coherent preference, since it is the result of an ambiguous deliberation and no ranking argument would be justified.
} 
this process allows us to move from the direct aggregation of agents' preferences to the aggregation of rational and justified sets of preferences (and their corresponding rankings).

More precisely, multiple extensions are computed (unless all the arguments coincide, i.e, having a consensus among the agents' preferences). Each extension contains the preference relation arguments and a single ranking argument which corresponds to a coherent aggregation of possible preference relations with their justifications. Hence, it is now possible to consider the extensions as (virtual) voters and aggregate their rankings. Given a semantics $s$, the set of justified preferences is denoted by $\mathcal{J P}^{s}$; hence, $\left|\mathcal{J P}^{s}\right|=\left|\boldsymbol{E}_{s} \backslash\left\{\mathcal{E} \in \boldsymbol{E}_{s}: \mathcal{A}_{B} \in \mathcal{E}\right\}\right|$, where $\boldsymbol{E}_{s}$ is the set of extensions obtained thanks to semantics $s$. We consider the ranking of each extension (except if the extension contains the blank vote) as a justified preference $\mathcal{J P}_{k}$, with $k \in\left[1,\left|\mathcal{J P}^{s}\right|\right]$. Each justified preference has preferences over the alternatives denoted with $x \succ \mathcal{J P}_{k} y$ which means that justified preference $\mathcal{J P}_{k}$ prefers alternative $x$ to $y$.

Informally, the collective justified preference profile is the set of all the justified preferences.

Definition[Justified pref. profile] A justified preference profile $\succ_{\mathcal{J P}} \mathcal{P}^{s}=$ $\left\langle\succ \mathcal{J P}_{1}, \ldots, \succ \mathcal{J P}_{\left|\mathcal{J P} \mathcal{P}^{s}\right|}\right\rangle \in \mathcal{L}_{A}^{\left|\mathcal{J P}^{s}\right|}$ is a collection of linear orders for all the justified preferences obtained thanks to a semantics $s$.

For instance, using the preferred semantics would produce a justified preference profile $\succ \mathcal{J P}^{p}$

Example 2 (cont.). We use the "preferred" acceptability semantics in order to compute the extensions and thus, the justified preference profile $\succ_{\mathcal{J P}^{p}}$. The set of extensions is

$$
\boldsymbol{E}=\left\{\begin{array}{l}
\left\{\mathcal{A}_{1}, \mathcal{A}_{2}, \mathcal{A}_{a b}, \mathcal{A}_{a b}^{\prime}, \mathcal{A}_{b c}, \mathcal{A}_{b c}^{\prime}, \mathcal{A}_{a c}, \mathcal{A}_{\mathcal{R}_{a b c}}\right\}, \\
\left\{\mathcal{A}_{1}, \mathcal{A}_{2}, \mathcal{A}_{b a}, \mathcal{A}_{b c}, \mathcal{A}_{b c}^{\prime}, \mathcal{A}_{a c}, \mathcal{A}_{\mathcal{R}_{b a c}}\right\}, \\
\left\{\mathcal{A}_{1}, \mathcal{A}_{2}, \mathcal{A}_{a b}, \mathcal{A}_{a b}^{\prime}, \mathcal{A}_{c b}, \mathcal{A}_{a c}, \mathcal{A}_{\mathcal{R}_{a c b}}\right\}, \\
\left\{\mathcal{A}_{1}, \mathcal{A}_{2}, \mathcal{A}_{b a}, \mathcal{A}_{c b}, \mathcal{A}_{a c}, \mathcal{A}_{B}\right\}
\end{array}\right\}
$$

We derive the justified preference profile

$$
\succ_{\mathcal{J P}}=\left\langle a \succ_{\mathcal{J P}} b \succ_{\mathcal{J P}_{1}} c, b \succ_{\mathcal{J P}_{2}} a \succ_{\mathcal{J P}_{2}} c, a \succ_{\mathcal{J} \mathcal{P}_{3}} c \succ_{\mathcal{J P}_{3}} b\right\rangle .
$$

The aggregation of the justified preference profile gives a as the Condorcet winner, while the original preference profile leaded to the voting paradox.

As noted before, the justified preference profile can have multiple justified preferences (extensions), so we refer to classical social theory for aggregating them and hence, the collective decision produced by our method is a ranking of the alternatives. As we are going to see in the next section, the construction of the justified preference profile allows to avoid the voting paradox when the Condorcet method is used to declare the winner.

There can be cases where the justified preference profile consists only of blank votes and hence, a full tie between the alternatives may exist. This may happen if the reasoning part does not affect the preference profile, i.e., the 
generic arguments are not present or mutually attack each other. Therefore, this phenomenon shows the importance of deliberation and reasoning on the preferences and how a structured justified profile can behave in a better way since it is cyclic-free. For instance, removing generic arguments $\mathcal{A}_{1}, \mathcal{A}_{2}$ from our example will lead to a full tie between the alternatives because of lack of reasoning on the preferences.

\section{Avoiding the Condorcet Paradox}

This section is devoted to showing that the qualitative approach using abstract argumentation presented in this paper avoids the Condorcet paradox. The reason for which the Condorcet paradox is avoided lies on the reasoning capabilities of the ranking-completion argumentation framework. This type of framework along with Dung's preferred semantics help us construct the justified preference profile. This profile can be seen as a type of structured preferences in which a collective, according to Condorcet's method, cyclic preference will not exist when arguments posed during the deliberation phase are taken into account to compute the framework.

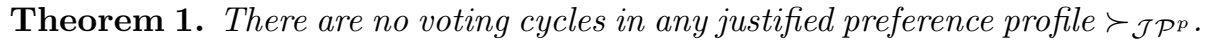

Proof. Please note that in the following, in order to avoid tedious notations, when we are referring to $\mathcal{J P}^{p}$ we are going to use the notation $\mathcal{J P}$.

Let $\left|\succ_{\mathcal{J P}}\right|=k$. In order to have a voting cycle, there must exist a justified preference profile $\succ \mathcal{J P}$ where we have three alternatives $x, y, z \in A$ such that the following conditions hold:

$$
\begin{array}{ll}
\mid \mathcal{J P}_{i} \in \succ_{\mathcal{J P}}: & x \succ_{\mathcal{J P}_{i}} y \mid>k / 2, \\
\mid \mathcal{J P}_{i} \in \succ_{\mathcal{J P}}: & y \succ_{\mathcal{J P}_{i}} z \mid>k / 2, \\
\mid \mathcal{J P}_{i} \in \succ_{\mathcal{J P}}: & z \succ_{\mathcal{J P}_{i}} x \mid>k / 2
\end{array}
$$

Let $\mathcal{R}_{u v}$ be the subset of the set of rankings $\mathcal{R}$ in which $u$ is ranked over $v$. Hence, all the possible sets of ranking arguments which are derived from $\mathcal{R}$ between these three alternatives are the following: $\mathbf{A}_{\mathcal{R}_{x y}}, \mathbf{A}_{\mathcal{R}_{y x}}$, $\mathbf{A}_{\mathcal{R}_{x z}}, \mathbf{A}_{\mathcal{R}_{z x}}, \mathbf{A}_{\mathcal{R}_{y z}}, \mathbf{A}_{\mathcal{R}_{z y}}$.

We are considering all the cases that may appear in the ranking-completion argumentation framework and as a consequence in the justified preference profile, with respect to these three alternatives and the preference relations arguments.

Case 1: When we have less than three preference relation arguments, we have two possible subcases. In the subcase where they can mutually attack each other, i.e., $\mathcal{A}_{x y}$ and $\mathcal{A}_{y x}$, it is easy to see that $x$ is over $y$ in half of the extensions rankings, and $y$ is over $x$ in the other half, which leads to a tie between $x$ and $y$. In the other subcase it is easy to see that we cannot have all the ranking 
arguments $\mathcal{A}_{\mathcal{R}_{x y z}}, \mathcal{A}_{\mathcal{R}_{y z x}}$ and $\mathcal{A}_{\mathcal{R}_{z x y}}$ in the extensions out of only two preference relation arguments and hence, their corresponding rankings. Therefore, in both cases we do not have a cycle.

Case 2: When we have three preference relation arguments then in order to have a cycle we need to have these three arguments $\mathcal{A}_{x y}, \mathcal{A}_{y z}$ and $\mathcal{A}_{z x}$ (or equivalently $\mathcal{A}_{y x}, \mathcal{A}_{z y}$ and $\mathcal{A}_{x z}$ ) to be included in the framework. This is valid because in order for alternative $x$ to be ranked over $y$ in the justified preference $\mathcal{J P}_{i}$, i.e., $x \succ \mathcal{J P}_{i} y$ we need that the preference relation argument $\mathcal{A}_{x y}$ or a set of arguments of the form $\mathcal{A}_{x \mathcal{T} y}$ to be included in the corresponding extension and hence, in the argumentation framework. The set $\mathcal{A}_{x \mathcal{T}}$ is the set of arguments that are transitively equivalent to $\mathcal{A}_{x y}$, i.e., $\mathcal{A}_{x \mathcal{T} y}=\left(\mathcal{A}_{x \omega} \cup \cdots \cup \mathcal{A}_{\psi y}\right), \omega, \psi \in$ $A$. However, if we include arguments of the form $\mathcal{A}_{x \mathcal{T} y}$ then we have more than three arguments. Therefore, $\mathcal{A}_{x y}$ must be included in the framework and similarly, $\mathcal{A}_{y z}$ and $\mathcal{A}_{z x}$ must also be included.

We will show now that the cycle can be avoided when we have these three or more arguments. If we have only these three arguments $\mathcal{A}_{x y}, \mathcal{A}_{y z}$ and $\mathcal{A}_{z x}$ included in the framework then all ranking arguments where $y \succ x, z \succ y$ and $x \succ z$ are attacked and thus, not included in the set of the extensions, i.e., the arguments of sets $\mathbf{A}_{\mathcal{R}_{y x}}, \mathbf{A}_{\mathcal{R}_{z y}}$ and $\mathbf{A}_{\mathcal{R}_{x z}}$. For the other possible ranking arguments, let us assume that the elements of $\mathbf{A}_{\mathcal{R}_{x y}}$ are included in the extensions. We can now distinguish between the following cases with regard to the rank of $z$ compared to $x$ and $y$.

- if $z \succ x \succ y$ then we have $z \succ y$ and hence, all the rankings in $\mathbf{A}_{\mathcal{R}_{z y}}$.

- if $x \succ z \succ y$ then we have $z \succ y$ and hence, all the rankings in $\mathbf{A}_{\mathcal{R}_{z y}}$.

- if $x \succ y \succ z$ then we have $x \succ z$ and hence, all the rankings in $\mathbf{A}_{\mathcal{R}_{x z}}$.

We can see that in all the cases where $x \succ y$ leads to arguments rankings that are not included in the extensions and hence, the elements of $\mathbf{A}_{\mathcal{R}_{x y}}$ are not included in the set of the extensions. Similarly, we can see that $\mathbf{A}_{\mathcal{R}_{z x}}$ and $\mathbf{A}_{\mathcal{R}_{y z}}$ are neither included in the set of extensions. Thus, the only ranking argument which is included in the extensions is the blank vote which leads in the avoidance of voting cycles.

The next step of the proof is to consider all the cases with respect to these three alternatives with the addition of the remaining preference relations arguments. We will also show that for the remaining cases the voting cycles are avoided.

Case 3: The following preference relation arguments are included in the framework: $\mathcal{A}_{x y}, \mathcal{A}_{y z}, \mathcal{A}_{z x}$ and $\mathcal{A}_{y x}$. The first extension we are computing contains arguments $\mathcal{A}_{x y}, \mathcal{A}_{y z}, \mathcal{A}_{z x}$ and the blank vote $\mathcal{A}_{B}$. All the other extensions will contain the preference relation arguments $\mathcal{A}_{y x}, \mathcal{A}_{y z}, \mathcal{A}_{z x}$ and the ranking arguments that are not attacked by them. Argument $\mathcal{A}_{y x}$ attacks the ranking arguments in $\mathbf{A}_{\mathcal{R}_{x y}}$, while $\mathcal{A}_{y z}$ and $\mathcal{A}_{z x}$ attack arguments in $\mathbf{A}_{\mathcal{R}_{z y}}$ and $\mathbf{A}_{\mathcal{R}_{x z}}$ respectively. Therefore, for arguments in $\mathbf{A}_{\mathcal{R}_{y x}}$ we distinguish as above between 
the following cases with regard to the rank of $z$ compared to $y$ and $x$. Similarly for the remaining non-attacked ranking arguments we distinguish with regard to the rank of $x$ compared to $y$ and $z$ and the rank of $y$ compared to $z$ and $x$. Then, the only set of ranking arguments that is not attacked is the set $\mathbf{A}_{\mathcal{R}_{y z x}}$ which corresponds to the subset of rankings $\mathcal{R}_{y z x}$, i.e., all the rankings where $y \succ z \succ x$. Therefore the justified preference profile is composed of the blank vote and $\mathcal{R}_{y z x}$, which leads to no cycles.

A similar approach can be used when the following preference relation arguments are included in the framework: $\mathcal{A}_{x y}, \mathcal{A}_{y z}, \mathcal{A}_{z x}$ and $\mathcal{A}_{z y}$ (or $\mathcal{A}_{x z}$ resp.). It is easy to see that the only set of ranking arguments that is not attacked is the set $\mathbf{A}_{\mathcal{R}_{z x y}}\left(\mathbf{A}_{\mathcal{R}_{x y z}}\right.$ resp.) which corresponds to the subset of rankings $\mathcal{R}_{z x y}$ $\left(\mathcal{R}_{x y z}\right.$ resp.), i.e., all the rankings where $z \succ x \succ y(x \succ y \succ z$ resp.). Therefore the justified preference profile is composed of the blank vote and $\mathcal{R}_{z x y}\left(\mathcal{R}_{x y z}\right.$ resp.), which leads to no cycles.

Case 4: The following preference relation arguments are included in the framework: $\mathcal{A}_{x y}, \mathcal{A}_{y z}, \mathcal{A}_{z x}, \mathcal{A}_{y x}$ and $\mathcal{A}_{z y}$. The first extension we are computing contains arguments $\mathcal{A}_{x y}, \mathcal{A}_{y z}, \mathcal{A}_{z x}$ and the blank vote $\mathcal{A}_{B}$. We have $k / 3$ extensions that contain the preference relation arguments $\mathcal{A}_{y x}, \mathcal{A}_{y z}, \mathcal{A}_{z x}$ and the ranking arguments that are not attacked by them. As in Case 3 , the only set of ranking arguments that is not attacked is the set $\mathbf{A}_{\mathcal{R}_{y z x}}$ which corresponds to the subset of rankings $\mathcal{R}_{y z x}$, i.e., all the rankings where $y \succ z \succ x$. We also have $k / 3$ extensions that contain the preference relation arguments $\mathcal{A}_{x y}, \mathcal{A}_{z y}, \mathcal{A}_{z x}$ and the ranking arguments that are not attacked by them. Then, the only set of ranking arguments that is not attacked is the set $\mathbf{A}_{\mathcal{R}_{z x y}}$ which corresponds to the subset of rankings $\mathcal{R}_{z x y}$, i.e., all the rankings where $z \succ x \succ y$. Finally, we have $k / 3$ extensions that contain the preference relation arguments $\mathcal{A}_{y x}, \mathcal{A}_{z y}$, $\mathcal{A}_{z x}$ and the ranking arguments that are not attacked by them. Then, the only set of ranking arguments that is not attacked is the set $\mathbf{A}_{\mathcal{R}_{z y x}}$ which corresponds to the subset of rankings $\mathcal{R}_{z y x}$, i.e., all the rankings where $z \succ y \succ x$. Therefore, in the justified preference profile we have the blank vote and $2 \cdot k / 3$ justified preferences where $z \succ y, z \succ x$ and $y \succ x$, which leads to no cycles.

A similar approach can be used when the following preference relation arguments are included in the framework: $\mathcal{A}_{x y}, \mathcal{A}_{y z}, \mathcal{A}_{z x}, \mathcal{A}_{x z}$ and $\mathcal{A}_{y x}$ (or $\mathcal{A}_{z y}$ resp.). Here also, we have one extension with $\mathcal{A}_{x y}, \mathcal{A}_{y z}, \mathcal{A}_{z x}$ and $\mathcal{A}_{B} ; k / 3$ extensions that contain the preference relation arguments $\mathcal{A}_{y x}$ ( $\mathcal{A}_{x y}$ resp.), $\mathcal{A}_{y z}\left(\mathcal{A}_{z y}\right.$ resp.), $\mathcal{A}_{z x}$ which correspond to all the rankings where $y \succ z \succ x(z \succ x \succ y$ resp.); $k / 3$ extensions that contain the preference relation arguments $\mathcal{A}_{x y}, \mathcal{A}_{y z}$, $\mathcal{A}_{x z}$ which correspond to all the rankings where $x \succ y \succ z ; k / 3$ extensions that contain the preference relation arguments $\mathcal{A}_{y x}\left(\mathcal{A}_{x y}\right.$ resp.), $\mathcal{A}_{y z}\left(\mathcal{A}_{z y}\right.$ resp.), $\mathcal{A}_{x z}$ which correspond to all the rankings where $y \succ x \succ z(x \succ z \succ y$ resp.). Hence, cycles are avoided since we have $2 \cdot k / 3$ justified preferences where $y \succ z, x \succ z$ and $y \succ x(z \succ y, x \succ z$ and $x \succ y$ resp.) and the blank vote.

Case 5: The last case is when all the preference relation arguments derived from $x, y, z$ are included in the framework. There are two extensions containing 
the blank vote $\mathcal{A}_{B}$, i.e., the one that contains the arguments $\mathcal{A}_{x y}, \mathcal{A}_{y z}, \mathcal{A}_{z x}$ and the symmetric one that contains $\mathcal{A}_{y x}, \mathcal{A}_{z y}, \mathcal{A}_{x z}$. Regarding the rest of the extensions we have symmetrical cases, i.e., we have $k / 6$ extensions containing the elements of $\mathbf{A}_{\mathcal{R}_{x y z}}$ and respectively $k / 6$ extensions for each one of the possible ranking arguments. In this case we have a full equivalence, which means that there is a tie between $x, y, z$.

Recall that, due to Axiom 1, a generic argument cannot have an impact on preferences over different alternatives and hence, cannot attack two different preference relations arguments nor a ranking argument. Therefore, a generic argument can attack or defend a single preference relation argument. The latter is done by attacking the generic argument who attacks the preference relation argument. Since a generic arguments is only related to one preference relation argument, this leads to the conclusion that the outcome is not affected even if we have possible additions or removals of preference relation arguments in the extensions. An attack (or defence) of a generic argument can only affect one specific preference relation argument and removing (or adding) it does not change the analysis since we are coming back to the same cases described above when the consideration of only the preference relation arguments is taken into account.

Concluding, in all the above considered possible cases we have justified preference profiles without voting cycles and thus, we have completed the proof of Theorem 1.

\section{Social Choice desirable properties}

In the current section we explore the behaviour of the proposed approach towards desirable properties from the viewpoint of Social Choice. The Condorcet paradox is not the only deficiency that many well-known voting rules have. In order to evaluate further the method we are also referring to classical desirable properties from the social point of view which are often not satisfied.

\subsection{Monotonicity}

In the following section, we present our results about the property of monotonicity for our proposed method. A system is monotonic if a winning alternative remains the winning one in the new profile created after she is moved upward in the preferences of some of the agents.

Theorem 2. The proposed method satisfies monotonicity when Condorcetconsistent voting rules are used for the aggregation of $\succ \mathcal{J P}$.

Proof. Suppose that $w$ is the winning alternative in the original instance of the problem, i.e., having as input the preference profile $\succ_{P P}$. If we raise $w$ in the preferences of some agents then we get as input the new profile $\succ_{P P}^{\prime}$. In this 
profile compared to the original one $\succ_{P P}$ some preference relations arguments have been added and some have been removed because of moving $w$ upwards.

For the analysis, we consider a reduced argumentation framework and the preference relation arguments without the justifications because it simplifies the proof and does not affect the result. Indeed, having two arguments $\mathcal{A}_{x y}$ and $\mathcal{A}_{x y}^{\prime}$ in the same extension leads to the same ranking (linear order) of the justified preference $\mathcal{J} \mathcal{P}_{k}$, where the linear order of $\mathcal{J P}_{k}$ belongs to the set $\mathcal{R}_{x y}$. Hence, let $A F\left(\succ_{P P}\right)$ be the argumentation framework built from profile $\succ_{P P}$ but containing only one preference relation argument $\mathcal{A}_{x y}$ for declaring that an alternative $x$ is preferred to $y$ by an agent no matter what is the justification for this preference $(x \succ y)$. We consider now two cases for the set $\mathbf{A}_{P R}^{*}$ of the preference relations arguments that have been added or removed in the $A F\left(\succ_{P P}^{\prime}\right)$ compared to $A F\left(\succ_{P P}\right)$.

The first case is the one where the arguments in set $\mathbf{A}_{P R}^{*}$ are contained in both the original $A F\left(\succ_{P P}\right)$ and the new framework $A F\left(\succ_{P P}^{\prime}\right)$. Observe that, this can happen because we consider the arguments without the justifications and if $\mathcal{A}_{w y}$ already exists adding $\mathcal{A}_{w y}^{\prime}$ with a new justification will not create a new argument but will point to the existing one $\left(\mathcal{A}_{w y}\right)$. In this case all the arguments point to existing ones and thus, the $A F\left(\succ_{P P}^{\prime}\right)$ equals to $A F\left(\succ_{P P}\right)$. Therefore, we compute the same extensions from both frameworks and hence, the new justified preference profile, i.e., $\succ_{\mathcal{J P}}^{\prime}$ is the same as the original one $\succ_{\mathcal{J P}}$. Consequently, the aggregation of both $\succ_{\mathcal{J P}}^{\prime}$ and $\succ_{\mathcal{J P}}$ gives the same outcome under any voting rule, hence, $w$ remains the winner.

In the second case, a subset of arguments in $\mathbf{A}_{P R}^{*}$ are not contained in both the frameworks. All the arguments of type $\mathcal{A}_{w y}$ should be included in both frameworks otherwise $w$ cannot be the Condorcet winner. Indeed if the arguments $\mathcal{A}_{w y}$ are not included in $A F\left(\succ_{P P}\right)$ or $A F\left(\succ_{P P}^{\prime}\right)$ then the ranking arguments $\mathbf{A}_{\mathcal{R}_{w \cdots y}}$ and thus, the rankings $\mathcal{R}_{w y}$ where $w$ is over $y$ cannot exist. Then, the only case we can have is to either add or remove the arguments $\mathcal{A}_{y w}$. If this subset of arguments is removed and not contained in the $A F\left(\succ_{P P}^{\prime}\right)$ then all rankings in $\succ_{\mathcal{J P}}^{\prime}$ where $y$ is over $w$ are also removed and thus, $w$ still beats $y$ in a pairwise comparison and remains the Condorcet winner. Finally, note that we cannot add the arguments $\mathcal{A}_{y w}$ since $A F\left(\succ_{P P}^{\prime}\right)$ is a preference profile where the winner $w$ is moved upwards in the preferences and not $y$.

Therefore taking into account all the cases regarding the differences between profiles $\quad A F\left(\succ_{P P}\right)$

$A F\left(\succ_{P P}^{\prime}\right)$, alternative $w$ remains the winner.

\subsection{Homogeneity}

In the following section, we present our results about the property of homogeneity for the proposed method. A system is homogeneous if the replication of the preference profile does not change the winning ranking of the alternatives. 
Theorem 3. The proposed method satisfies homogeneity for any voting rule used for the aggregation of $\succ \mathcal{J P}$.

Proof. Suppose that $\mathcal{R}$ is the winning ranking in the original instance of the problem, i.e., having as input the preference profile $\succ_{P P}$. If we replicate the original profile by $n$ times then we get as input the new profile $\succ_{P P}^{n}$. It is easy to see that this profile produces the exact same set of preference relations arguments when compared to the original one. Hence, the argumentation framework built from it, i.e. $A F\left(\succ_{P P^{n}}\right)$, is the same as the original one $A F\left(\succ_{P P}\right)$ and thus, the new justified preference profile, $\succ_{\mathcal{J P}}^{n}$, is the same as the original one, $\succ_{\mathcal{J P}}$. Consequently, the aggregation of both $\succ_{\mathcal{J} \mathcal{P}}^{n}$ and $\succ_{\mathcal{J P}}$ gives the same outcome under any voting rule and thus, the winning ranking $\mathcal{R}$ remains the same under both profiles.

\section{Conclusion and future work}

In this paper, we have proposed a framework for decision-making that relies on the qualitative preferences which comes in contrast with the social choice methods which rely only on the quantitative aggregation of the individual preferences. The intuition behind our approach was to find a method that simulates a pre-voting, i.e., deliberation, phase in a collective decision and that takes into account the agents' expressed arguments. Hence, the proposed method produces arguments based on the preferences of the agents and the justifications behind these preferences. The aggregation of all the agents' arguments is computed based on the attacks between them and leads to possible extension(s) where each one of them depicts collectively justified preferences. When there is no consensus among agents' preferences, multiple extensions are computed and hence, a Condorcet method can be used to aggregate the collective justified preference profile. Due to the construction of the argumentation framework, the justified preference profile is a type of structured profile that can avoid the Condorcet paradox.

In terms of future work, we want to further extend our research towards the collective multi-criteria decision-making problem and get deeper in the integration of argumentation with computational social choice. We plan to explore techniques from computational social choice that will permit us to propose decision aiding procedures that can support group preferences and we believe that argumentation framework can provide us the reasoning behind the decision makers preferences. The combination of these two subfields of computer science will allow us to explain the decisions rationally and thus, we want to propose more procedures for collective decisions that will have more chances to be accepted by the society. To strengthen this view we plan to propose quantitative methods that can compare and evaluate the different decision-making procedures. Also, it is left for future work to study the complexity of our approach; in general abstract argumentation is subject to quite bad complexities (see for instance 
[16] and [17]), but solvers such as CoQuiAAS ${ }^{3}$ might allow to handle quite large argumentation systems as shown in [31]. Therefore, it would be interesting to adapt this kind of solvers to the ranking-completion argumentation framework in order to assess the practical relevance of our approach.

\section{Acknowledgement}

The research was implemented with a scholarship from IKY funded by the action "Support of Postdoctoral Researchers" from the resources of the EP "Human Resources Development, Education and Lifelong Learning" with priority axes 6, 8, 9 and is co-funded by the European Social Fund-ESF and the Greek state.

This publication has been written with the support of the AgreenSkills + fellowship programme which has received funding from the EU's Seventh Framework Programme under grant agreement No FP7-609398 (AgreenSkills+ contract).

\section{References}

[1] S. Airiau, E. Bonzon, U. Endriss, N. Maudet, and J. Rossit. Rationalisation of profiles of abstract argumentation frameworks. In Proceedings of the 2016 International Conference on Autonomous Agents \& Multiagent Systems (AAMAS '16), pages 350-357, 2016.

[2] L. Amgoud, J.-F. Bonnefon, and H. Prade. An argumentation-based approach to multiple criteria decision. In Proceedings of the 8th European Conference on Symbolic and Quantitative Approaches to Reasoning and Uncertainty (ECSQARU '05), pages 269-280, 2005.

[3] L. Amgoud and H. Prade. Using arguments for making and explaining decisions. Artificial Intelligence, 173(3-4):413-436, 2009.

[4] K. Arrow and H. Raynaud. Social choice and multicriterion decision-making. MIT Press, Cambridge, 1986.

[5] K. J. Arrow. A difficulty in the concept of social welfare. Journal of Political Economy, 58(4):328-346, 1950.

[6] R. Benayoun, B. Roy, and B. Sussman. ELECTRE: une méthode pour guider le choix en présence des points de vue multiples. Note de travail 49. Technical report, SEMA-METRA International, Direction Scientifique, 1966.

[7] P. Besnard and A. Hunter. Elements of Argumentation. MIT Press, 2008.

[8] D. Black. Theory of Committees and Elections. Cambridge University Press, 1958.

\footnotetext{
${ }^{3}$ http://www.cril.univ-artois.fr/coquiaas/
} 
[9] G. Bodanza, F. Tohmé, and M. Auday. Collective argumentation: A survey of aggregation issues around argumentation frameworks. Argument $\&$ Computation, 8(1):1-34, 2017.

[10] J.-F. Bonnefon and H. Fargier. Comparing sets of positive and negative arguments: Empirical assessment of seven qualitative rules. In Proceedings of the 2006 Conference on ECAI 2006: 17th European Conference on Artificial Intelligence, pages 16-20, 2006.

[11] F. Brandt. Some remarks on Dodgson's voting rule. Mathematical Logic Quarterly, 55(4), 460-463, 2009.

[12] F. Brandt, V. Conitzer, U. Endriss, J. Lang, and A. D. Procaccia. Handbook of Computational Social Choice. Cambridge University Press, New York, NY, USA, 1st edition, 2016.

[13] M. D. Condorcet. Essai sur l'application de l'analyse à la probabilité de décisions rendues à la pluralité de voix. Imprimerie Royal. Facsimile published in 1972 by Chelsea Publishing Company, New York., 1785.

[14] F. Dietrich, C. List. A Reason-Based Theory of Rational Choice Noûs, 47(1):104-134, 2011

[15] P. M. Dung. On the acceptability of arguments and its fundamental role in nonmonotonic reasoning, logic programming and n-person games. Artificial intelligence, $77(2): 321-357,1995$.

[16] P. E. Dunne, M. Wooldridge. Complexity of Abstract Argumentation. Argumentation in Artificial Intelligence, pages 85-104, 2009.

[17] W. Dvorák, S Woltran Complexity of semi-stable and stage semantics in argumentation frameworks. Inf. Process. Lett., 110(11):425-430, 2010.

[18] D. S. Felsenthal and N. Tideman. Varieties of failure of monotonicity and participation under five voting methods. Theory and Decision, 75(1):59-77, 2013.

[19] P. C. Fishburn. Monotonicity paradoxes in the theory of elections. Discrete Applied Mathematics, Volume 4, Issue 2, 119-134, 1982.

[20] J. Fox and S. Parsons. On using arguments for reasoning about actions and values. In Proceedings of the AAAI Spring Symposium on Qualitative Preferences in Deliberation and Practical Reasoning, pages 55-63, 1997.

[21] A. Gibbard. Manipulation of voting schemes: A general result. Econometrica, 41(4):587-601, 1973.

[22] N. I. Karacapilidis and D. Papadias. A group decision and negotiation support system for argumentation based reasoning. In Selected Papers from the Workshop on Reasoning with Incomplete and Changing Information and on Inducing Complex Representations(PRICAI'96), pages 188-205, 1998. 
[23] R. L. Keeney and H. Raiffa. Decisions with multiple objectives: Preferences and value tradeoffs. Wiley, New York, 1976.

[24] M. Morge and P. Mancarella. The hedgehog and the fox. an argumentationbased decision support system. In Proceedings of the 4th International Workshop on Argumentation in Multi-Agent Systems (ArgMas '07), pages 55-68, 2007.

[25] A. Ostanello. Outranking Methods, pages 41-60. Springer Berlin Heidelberg, Berlin, Heidelberg, 1985.

[26] B. Roy. Classement et choix en présence de points de vue multiples (la méthode electre). La Revue d'Informatique et de Recherche Opérationelle (RIRO), (8):57-75, 1968.

[27] B. Roy. The outranking approach and the foundations of electre methods. Theory and Decision, 31(1):49-73, 1991.

[28] B. Roy and D. Bouyssou. Aide multicritère à la décision: Méthodes et cas. Economica, 1993.

[29] M. A. Satterthwaite. Strategy-proofness and arrow's conditions: Existence and correspondence theorems for voting procedures and social welfare functions. Journal of Economic Theory, 10(2):187 - 217, 1975.

[30] D. V. Winterfeldt and W. Edwards. Decision analysis and behavorial research. Cambridge University Press, Cambridge., 1986.

[31] B. Yun, S. Vesic, M. Croitoru, P. Bisquert, R. Thomopoulos. A Structural Benchmark for Logical Argumentation Frameworks. Advances in Intelligent Data Analysis XVI, pages 334-346, 2017. 